\title{
Radiographic and clinical outcome of lumbar lateral interbody fusion for extreme lumbar spinal stenosis of Schizas grade D: a retrospective study
}

Jun Li

Zhejiang University School of Medicine Second Affiliated Hospital

Hao Li

Zhejiang University School of Medicine Second Affiliated Hospital

Zhang Ning zhejiang university school of medicine second affliated hospital

Zhi-wei Wang

Zhejiang University School of Medicine Second Affiliated Hospital

\section{Teng-fei Zhao}

Zhejiang University School of Medicine Second Affiliated Hospital

Lin-wei Chen

Zhejiang University School of Medicine Second Affiliated Hospital

\section{Gang Chen}

Zhejiang University School of Medicine Second Affiliated Hospital

\section{Qi-xin Chen}

Zhejiang University School of Medicine Second Affiliated Hospital

Fang-cai Li ( $\sim 2505004 @ z j u . e d u . c n)$

Spine Surgery, Second Affiliated Hospital, School of Medicine, Zhejiang University https://orcid.org/0000-0002-2402-781X

\section{Research article}

Keywords: LLIF; lumbar; spinal stenosis; indirect decompression; radiographical outcomes; clinical outcomes

Posted Date: February 12th, 2020

DOI: https://doi.org/10.21203/rs.2.23266/v1

License: (c) (i) This work is licensed under a Creative Commons Attribution 4.0 International License. Read Full License 
Version of Record: A version of this preprint was published at BMC Musculoskeletal Disorders on April 20th, 2020. See the published version at https://doi.org/10.1186/s12891-020-03282-6. 


\section{Abstract}

Background: Extreme lumbar spinal stenosis was thought to be a relative contraindication for lumbar lateral interbody fusion (LLIF) and was excluded in most studies. This is a retrospective study to analyze the radiographic and clinical outcome of LLIF for extreme lumbar spinal stenosis of Schizas grade D.

Methods: For radiographic analysis, we included 202 segments from 124 patients who underwent LLIF between June 2017 and December 2018. Lumbar spinal stenosis was graded according to Schizas' classification. Anterior and posterior disc heights, disc angle, foramen height, spinal canal diameter and central canal area were measured on CT and MRI. For clinical analysis, 18 patients with at least one segment of grade D were included. Visual analogue scale (VAS) and Oswestry disability index (ODI) scores were used to evaluate clinical outcome. Continuous variables were confronted by using Student's t-test, obtaining a statistically significant difference for values inferior to 0.05 .

Results: Among the 202 segments included for radiological evaluation, there were 42 grade A segments, 41 grade $B$ segments, 101 grade $C$ segments and 18 grade D segments. Postoperatively, the average change of midsagittal canal diameter of grade $D$ was significantly greater than that of grade $A$, and not significantly different compared to grades $B$ and $C$. As to the average changes of disc height, bilateral foraminal height, disc angle and central canal area (CCA), grade $D$ was not significantly different from the others. The average postoperative CCA of grade D was significantly smaller than the average preoperative CCA of grade C. Eighteen patients with grade D stenosis were followed up for an average of $19.61 \pm 6.32$ months. Clinical evaluation revealed an average improvement in the ODI and VAS scores for back and leg pain by $20.77 \%, 3.67$ and 4.15 points, respectively. Sixteen of 18 segments with grade $D$ underwent posterior decompression.

Conclusion: The radiographic decompression effect of LLIF for Schizas grade D segments were comparable with that of other grades. Posterior decompression was necessary for LLIF to achieve a satisfactory clinical outcome for extreme lumbar spinal stenosis of Schizas grade D.

\section{Background}

As a minimally-invasive technique, lateral lumbar interbody fusion (LLIF) has become the first choice of many spine surgeons in recent years. LLIF is capable of restoring foraminal and intervertebral height, thecal sac area, and alignment while with less trauma and lower approach-related morbidity compared with traditional open decompression techniques $(1,2)$, making it especially suitable for elderly patients, patients with multi-level lumbar spine diseases and patients who cannot tolerate large operations.

LLIF, as an indirect decompression technique, does not directly remove a disc or osteophyte protruding into the spinal canal, and its decompression effect is not as thorough as traditional posterior decompression surgery. Radiographic studies have shown that improvement of the cross-sectional area of the spinal canal is significantly smaller after LLIF than after minimally-invasive transforaminal lumbar interbody fusion $(3,4)$. Generally, extreme central canal stenosis, defined by a complete loss of 
cerebrospinal fluid signal on preoperative magnetic resonance imaging (MRI), was thought to be a relative contraindication for LLIF. According to Schizas' classification(5), Grade D stenosis is defined as extreme stenosis, in which, in addition to no rootlets being recognizable, there is no epidural fat posterior to the dural sac (Fig. 1). Since patients with extreme stenosis (grade D) were excluded in most studies, the clinical and radiographic outcomes of LLIF for extreme lumbar spinal stenosis remain unknown. However, extreme lumbar spinal stenosis is common in clinical practice, especially in patients with multilevel lumbar degenerative disease. For the sake of less invasiveness, it is reasonable to perform LLIF for those patients instead of traditional open surgery, although additional posterior decompression is sometimes needed. The current study was the first to evaluate the indirect neural decompression effect in patients with extreme lumbar spinal stenosis. In current study, we compared the radiographic outcomes of LLIF for stenosis of Schizas grades A, B, C and D. Then, clinical outcomes of LLIF for a series of cases with stenosis of Schizas grade $\mathrm{D}$ were retrospectively evaluated.

\section{Methods}

\section{Patients}

Retrospectively, patients with the main diagnosis of degenerative lumbar spinal stenosis who underwent crenel lateral interbody fusion (CLIF) $(6,7)$, a modified extreme lateral interbody fusion technique, by our surgical group between June 2017 and December 2018 were reviewed. Patients who suffered from significant lumbar scoliosis, grade 2 spondylolisthesis, lumbar fracture or had undergone prior lumbar surgery were excluded from this study. All the segments were grouped according to Schizas' lumbar stenosis classification(5). Grade A stenosis is the mildest, with abundant cerebrospinal fluid inside the dural sac. In grade B stenosis, the rootlets occupy the whole of the dural sac, but they can still be individualized. In grade $\mathrm{C}$, no rootlets can be recognized but epidural fat can be visualized posteriorly. In grade $D$, in addition to no rootlets being recognizable there is no epidural fat posteriorly.

\section{Radiological and clinical assessments}

Standing lateral plain radiographs, MRI, and CT scans were obtained for all patients preoperatively and

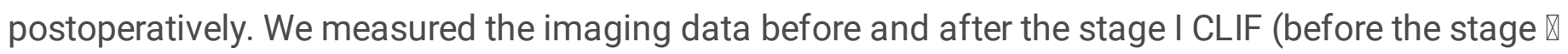
posterior internal fixation). All radiographic parameters were measured using measurement tools on PACS. The main measurement indexes included: the disc angle (DA), the anterior and posterior disk height (ADH and PDH), the bilateral intervertebral foramen height (IFH) on CT, and midsagittal canal diameter (CD) and the axial central canal area (CCA) on MRI (Fig.2).

A total of 18 patients with at least one level with grade $D$ stenosis who were followed for at least 6 months were clinically reviewed. The patients comprised seven males and eight females with a follow-up time of $18.53 \pm 6.39$ months (range: $9-26$ months) (Tab.1). Clinical outcomes were assessed by an experienced clinical research coordinator using a visual analogue scale (VAS) for back and leg pain and 
Oswestry Disability Index (ODI). The minimal clinically important difference for the ODI was 10 points(8). These data were compared between before surgery and at the last follow-up. In addition, perioperative data and complications were recorded.

\section{Surgical techniques}

The CLIF technique is a modified technique of lateral lumbar interbody fusion, aimed to minimize the approach-related complications of the traditional transpsoas approach (XLIF)(6). There are some unique features different from traditional XLIF. The psoas muscle working window was selected according to safe working zone on axial MRI of the target intervertebral space, the sagittal central line of the working zone should be located at least $1 \mathrm{~cm}$ anterior to nerve root. The psoas muscle was split longitudinally along the muscle fiber until the lateral intervertebral space was visualized. The genitofemoral nerve inside the psoas muscle might be found and was gently mobilized to posterior with a small amount of muscle fiber. A novel designed retractor was positioned in longitudinal direction to maintain the working window of psoas muscle. Two vertebral screws were used to fix the retractors to the vertebral body as close as possible to the endplate, and then assemble the retractors to the fixed ring. The intervertebral space preparation and implant placement were consistent with the traditional LLIF.

During the second stage, usually one week after the first stage, additional direct posterior decompression was performed due to inadequate resolution of stenotic symptoms and the surgeons' preference. If direct decompression was required, open pedicle screws were applied, otherwise bilateral percutaneous screws were used.

\section{Statistics}

Descriptive data are represented as means \pm standard deviation (SD). Continuous variables were analyzed by 2-sample t test and paired t test. The data collected were processed using PASW Statistics 18.0 (SPSS Inc., Chicago, IL, USA). Values of $P<.05$ were considered to indicate statistical significance.

\section{Results}

\section{Comparison of radiographic outcomes with other grades}

Among the 202 segments included in this study, there were 42 (20.79\%) segments of grade A, 41 (20.30\%) segments of grade B, 101 (50\%) segments of grade $C$ and 18 (8.91\%) segments of grade $D$. Overall, both the average ADH and PDH were significantly increased (Table 2). Since the average preoperative PDH was significantly smaller than the $A D H$, the average change rate of PDH $(58.80 \pm$ $67.49 \%)$ was significantly larger than that of the ADH (34.44 $\pm 4.17 \%)$. The average increase of DA was $1.23 \pm 3.81^{\circ}(P<0.001)$, which is small, partly attributed to the greater improvement rate of PDH than $\mathrm{ADH}$. Both the average left and right IFH were significantly increased. The average change and change 
rate of right IFH were insignificantly greater than the left. The average midsagittal CD and axial CCA on MRI were significantly increased. The average change rate of midsagittal CD was $35.86 \pm 64.01 \%$. The average change rate of axial CCA was $25.97 \pm 26.06 \%$.

With regard to the average change of midsagittal $C D$, the change in grade $D$ was significantly greater than that in grade $A$, but did not differ significantly from grades B or C. Interestingly, the average change rate of midsagittal $C D$ increased from grade $A$ to $D$, peaking at $79.69 \pm 86.23 \%$. As to the change of axial CCA, grade $D$ did not differ significantly from the others. Likewise, the average change rate of axial CCA increased from grade $A$ to $D$, peaking at $52.91 \pm 34.41 \%$ (Table 3 and Fig. 3 ). With regard to the average change of $A D H, P D H, D A$ and both sides IFH, grade D showed no significant difference compared with the others (Table 4).

\section{Clinical Outcome Of Patients With Extreme Degenerative Lumbar Stenosis}

Eighteen patients with at least one level of grade D who underwent CLIF were clinically reviewed. All of them were retrospectively followed-up, with a mean follow-up time of $19.61 \pm 6.32$ months. The clinical follow-up analysis revealed a statistically-significant improvement of established outcome scores. The mean ODI improved from $43.33 \pm 7.32 \%$ preoperatively to $22.56 \pm 8.63 \%$ at the last follow-up $(P<0.005)$. In a similar manner, the VAS for back decreased from $6.06 \pm 1.35$ to $2.39 \pm 0.78(P<0.005)$, while the VAS for leg decreased from $5.39 \pm 1.24$ to $1.89 \pm 1.02$ ( $P<0.005)$. Sixteen of 18 segments $(88.89 \%)$ with grade $\mathrm{D}$ underwent posterior decompression (Figs. 4 and 5). Only one patient presented with worsening back pain and neurological function at 3 months after surgery, who received a stand-alone CLIF surgery. However, he refused to undergo a posterior decompression.

In this group, seven patients (38.89\%) presented with surgery-related complications. A total of 11 complications occurred in seven patients. Pain in the front of the thigh was reported in five cases, and numbness was observed in three cases. Muscle weakness of the psoas major muscle was decreased in two cases, one of them suffered from psoas hematoma and was relieved after conservative treatment (Fig. 6). One patient suffered from deep venous thrombosis and interventional therapy was performed. There were no complications such as knee extension weakness, vascular injury, sympathetic nerve injury, visceral injury or ureteral injury in this series.

\section{Discussion}

The current study shows that the radiographic decompression effect of LLIF for Schizas grade D segments was comparable with the effect on other grades. However, patients with extreme lumbar spinal stenosis are not good candidates for LLIF alone. Stand-alone LLIF is not suggested for such patients, but with concomitant posterior decompression, LLIF can achieve a satisfactory clinical outcome for extreme lumbar spinal stenosis. 
Although the indirect neural decompression effect of LLIF for lumbar stenosis has been addressed in previous studies $(3,4,9-13)$, the current study was the first to evaluate the indirect neural decompression effect in patients with extreme lumbar spinal stenosis. The average changes in CD and CCA of grade $D$ were $2.50 \pm 1.25 \mathrm{~mm}$ and $16.11 \pm 10.70 \mathrm{~mm} 2$, which was comparable with the effects in other grades, which indicated that the indirect decompression effect is not compromised in patients with extreme spinal stenosis. However, the average rates of change CD and CCA of grade D were $79.69 \pm 86.23 \%$ and $52.91 \pm 34.41 \%$ respectively, both of which increased from grade A to D (Figure.3B), and in accordance with Fujibayashi's finding(14) that the greater the stenosis preoperatively, the greater the improvement rate in neural decompression with LLIF compared with milder stenosis. Oliviera et al(11) described increases of $2.4 \mathrm{~mm}(33.1 \%)$ and $12.4 \mathrm{~mm}^{2}(8.4 \%)$, respectively, in central canal diameter and canal area after XLIF. Elowitz et al(15) found a $3.8 \mathrm{~mm}$ improvement in anterior-posterior diameter of the dural sac and the area of the dural sac increased an average of $83 \mathrm{~mm}^{2}(143 \%)$ after XLIF. Castellvi et al(12) found that the central canal area was improved by $10 \mathrm{~mm}(27 \%)$ and $23 \mathrm{~mm}^{2}(17 \%)$ at 3 months and 1 year, respectively, after XLIF. At the 3-month follow-up, Isaacs et al(3) found an increase in the central canal area of $20.8 \mathrm{~mm}^{2}$ and in the central canal diameter of $1.2 \mathrm{~mm}$ after XLIF. Except for the results reported by Elowitz et al., the improvement of central canal stenosis in patients with Schizas grade $D$ is comparable with those studies $(3,11,12)$.

With regard to the indirect decompression effect on foraminal stenosis, Oliviera et al(11) described an increase of $2.48 \mathrm{~mm}(13.1 \%)$ of foraminal height after XLIF. In another retrospective study with 90 patients undergoing LLIF, Alimi et al(16) found foraminal height increased by $3.1 \mathrm{~mm}$ (20\%). At 3-month follow-up, Isaacs et al(3) found an increase in the approach-side foraminal height of $2.16 \mathrm{~mm}$ and in the contralateral-side foraminal height of $1.39 \mathrm{~mm}$ after XLIF. For segments with Schizas grade $\mathrm{D}$, we found an increase of foraminal height of $2.11 \pm 1.75 \mathrm{~mm}(12.20 \pm 10.28 \%)$ on the left -side and $2.05 \pm 1.83 \mathrm{~mm}$ $(11.83 \pm 10.82 \%)$ on right-side, which is comparable with those studies and not significantly different from the improvement seen with other grades. Likewise, as to the change of anterior and posterior disk height and segment angle, grade D showed no significant difference from other grade. Thus, we consider that preoperative central canal stenosis does not significantly influence the degree of change of indirect decompression after LLIF.

In our group, the mean ODI and VAS scores were both significantly improved at the last follow-up. Postoperatively, the average axial CCA of grade D was $49.87 \pm 18.81 \mathrm{~mm}^{2}$, which was significantly smaller than the average preoperative axial CCA of grade C $\left(82.06 \pm 26.97 \mathrm{~mm}^{2}\right)$. Sixteen of $18(88.89 \%)$ segments with stenosis of grade $\mathrm{D}$ received posterior direct decompression. We believed that additional posterior decompression after LLIF was important to ensure sufficient decompression in patients with extreme lumbar spinal stenosis. In addition, it is extremely dangerous to perform posterior instrumentation without direct decompression in patients with severe stenosis exhibiting preoperative paralysis(17). The clinical indications for posterior decompression after LLIF were inconsistent. There have been studies which claimed that factors likely to cause failure of indirect decompression include cage subsidence, low bone mineral density, severe central canal stenosis, ligamentum flavum 
hypertrophy, and osteophytes in the lateral recess and foraminal canal $(11,14,18-22)$. Among them, severe central canal stenosis might be the major risk factor. Nakashima et al(17) claimed that patients with preoperative lower limb paralysis and severe stenosis were at a higher risk of perioperative neurological deterioration and that this was particularly true for patients exhibiting ligament ossification around the spinal canal. Moreover, factors that are less likely to influence indirect decompression in LLIF are cage position, cage type, side of approach, preoperative sagittal/coronal alignment, presence of facet arthropathy, spinal level (upper or lower lumbar spine), and number of operated spinal levels (9, 21, 2326).

In the current group, seven out of eight patients with extreme lumbar stenosis who underwent single-level LLIF received second-stage posterior decompression. One patient who was treated with single-level standalone LLIF reported worse of back pain and neurological deterioration at the last follow-up. Lack of posterior supplemental fixation may lead to a loss of acquired indirect decompression after the operation. Thus, we do not suggest stand-alone surgery for patients with extreme spinal stenosis. Posterior lumbar interbody fusion may be a better surgical option for patients with single-level extreme lumbar spinal stenosis.

There are some limitations to this study including the retrospective nature of the study, the limited followup, and the small sample size of grade D. Since CT and MRI were not performed during follow-up in most cases, we could not show the radiographic changes during follow-up. The clinical outcomes of patients with extreme lumbar stenosis were not compared with those with mild lumbar stenosis.

\section{Conclusions}

The radiographic decompression effect of LLIF for Schizas grade D segments were comparable with that of other grades. Patients with extreme lumbar spinal stenosis are not good candidates for LLIF alone. Posterior decompression was necessary for LLIF to achieve a satisfactory clinical outcome for extreme lumbar spinal stenosis of Schizas grade D.

\section{Abbreviations}

LLIF:Lumbar Lateral Interbody Fusion; CLIF:Crenel Lateral Interbody Fusion; MRI:Magnetic Resonance Imaging; ADH:Anterior Disk Height; PDH:Posterior Disk Height; DA:Disc Angle; IFH:Intervertebral Foramen Height; CD:Canal Diameter; CCA:Central Canal Area; ODI:Oswestry Disability Index; VAS:Visual Analogue Scale.

\section{Declarations}

\section{Acknowledgements}

The authors thank Dr. Meng-ling Tang for her useful assistance in statistical analysis. We thank International Science Editing for editing this manuscript. 


\section{Authors' contributions}

FL, QC and GC made a substantial contribution to the study design. HL, NZ, LC and ZW made a substantial contribution to the data analysis and interpretation. TZ made a substantial contribution to the statistical analysis. JL made a substantial contribution towards writing up the manuscript. All authors have read and approved the final manuscript.

\section{Funding}

This work is supported by National Natural Science Foundation of China $(81702225,81702220)$ and Major Scientific and Technological Plan for Medicine and Health of Zhejiang Province (WKJ『ZJ『1903) for language editing, statistical analysis and publication charge.

\section{Availability of data and materials}

The datasets used and/or analysed during the current study are available from the corresponding author on reasonable request.

\section{Ethics approval and consent to participate}

Ethics approval was obtained from the Second Affiliated Hospital, School of Medicine, Zhejiang University Human Research Ethics Committee (Reference:2019-527).

\section{Consent for publication}

Not applicable.

\section{Competing interests}

The Authors have no conflicts of interest to declare.

\section{References}

1. McAfee PC, Regan JJ, Geis WP, Fedder IL. Minimally invasive anterior retroperitoneal approach to the lumbar spine. Emphasis on the lateral BAK. Spine. 1998;23(13):1476-84.

2. Ozgur BM, Aryan HE, Pimenta L, Taylor WR. Extreme Lateral Interbody Fusion (XLIF): a novel surgical technique for anterior lumbar interbody fusion. The spine journal : official journal of the North American Spine Society.6(4):435-43.

3. Isaacs RE, Sembrano JN, Tohmeh AG. Two-Year Comparative Outcomes of MIS Lateral and MIS Transforaminal Interbody Fusion in the Treatment of Degenerative Spondylolisthesis: Part II: Radiographic Findings. Spine. 2016;41 Suppl 8:S133-44.

4. Lin GX, Akbary K, Kotheeranurak V, Quillo-Olvera J, Jo HJ, Yang XW, et al. Clinical and Radiologic Outcomes of Direct Versus Indirect Decompression with Lumbar Interbody Fusion: A Matched-Pair 
Comparison Analysis. World neurosurgery. 2018;119:e898-e909.

5. Schizas C, Theumann N, Burn A, Tansey R, Wardlaw D, Smith FW, et al. Qualitative grading of severity of lumbar spinal stenosis based on the morphology of the dural sac on magnetic resonance images. Spine. 2010;35(21):1919-24.

6. Zhengkuan X, Qixin C, Gang C, Fangcai L. The technical note and approach related complications of modified lateral lumbar interbody fusion. Journal of clinical neuroscience : official journal of the Neurosurgical Society of Australasia. 2019;66:182-6.

7. Xu Z, Li F, Chen G, Chen Q. Reassessment System and Staged Surgical Strategy with Minimally Invasive Techniques for Treatment of Severe Adult Spinal Deformities. World neurosurgery. 2019;126:e860-e8.

8. Hägg $\mathrm{O}$, Fritzell P, Nordwall A. The clinical importance of changes in outcome scores after treatment for chronic low back pain. European spine journal : official publication of the European Spine Society, the European Spinal Deformity Society, and the European Section of the Cervical Spine Research Society. 2003;12(1):12-20.

9. Park SJ, Lee CS, Chung SS, Kang SS, Park HJ, Kim SH. The Ideal Cage Position for Achieving Both Indirect Neural Decompression and Segmental Angle Restoration in Lateral Lumbar Interbody Fusion (LLIF). Clinical spine surgery. 2017;30(6):E784-E90. Epub 2016/06/29.

10. Tessitore E, Molliqaj G, Schaller K, Gautschi OP. Extreme lateral interbody fusion (XLIF): A singlecenter clinical and radiological follow-up study of 20 patients. Journal of clinical neuroscience : official journal of the Neurosurgical Society of Australasia. 2017;36:76-9.

11. Oliveira L, Marchi L, Coutinho E, Pimenta L. A radiographic assessment of the ability of the extreme lateral interbody fusion procedure to indirectly decompress the neural elements. Spine. 2010;35(26 Suppl):S331-7.

12. Castellvi AE, Nienke TW, Marulanda GA, Murtagh RD, Santoni BG. Indirect decompression of lumbar stenosis with transpsoas interbody cages and percutaneous posterior instrumentation. Clinical orthopaedics and related research. 2014;472(6):1784-91.

13. Sato J, Ohtori S, Orita S, Yamauchi K, Eguchi Y, Ochiai N, et al. Radiographic evaluation of indirect decompression of mini-open anterior retroperitoneal lumbar interbody fusion: oblique lateral interbody fusion for degenerated lumbar spondylolisthesis. European spine journal : official publication of the European Spine Society, the European Spinal Deformity Society, and the European Section of the Cervical Spine Research Society. 2017;26(3):671-8.

14. Fujibayashi S, Hynes RA, Otsuki B, Kimura H, Takemoto M, Matsuda S. Effect of indirect neural decompression through oblique lateral interbody fusion for degenerative lumbar disease. Spine (Phila Pa 1976). 2015;40(3):E175-82. Epub 2014/11/14.

15. Elowitz EH, Yanni DS, Chwajol M, Starke RM, Perin NI. Evaluation of indirect decompression of the lumbar spinal canal following minimally invasive lateral transpsoas interbody fusion: radiographic and outcome analysis. Minimally invasive neurosurgery : MIN. 2011;54(5-6):201-6. 
16. Alimi M, Hofstetter CP, Cong GT, Tsiouris AJ, James AR, Paulo D, et al. Radiological and clinical outcomes following extreme lateral interbody fusion. Journal of neurosurgery Spine. 2014;20(6):62335. Epub 2014/04/08.

17. Nakashima H, Kanemura T, Satake K, Ishikawa Y, Ouchida J, Segi N, et al. Unplanned Second-Stage Decompression for Neurological Deterioration Caused by Central Canal Stenosis after Indirect Lumbar Decompression Surgery. Asian spine journal. 2019:584-91.

18. Lang G, Perrech M, Navarro-Ramirez R, Hussain I, Pennicooke B, Maryam F, et al. Potential and Limitations of Neural Decompression in Extreme Lateral Interbody Fusion-A Systematic Review. World neurosurgery. 2017;101:99-113.

19. Wang TY, Nayar G, Brown CR, Pimenta L, Karikari IO, Isaacs RE. Bony Lateral Recess Stenosis and Other Radiographic Predictors of Failed Indirect Decompression via Extreme Lateral Interbody Fusion: Multi-Institutional Analysis of 101 Consecutive Spinal Levels. World neurosurgery. 2017;106:819-26.

20. Malham GM, Parker RM, Goss B, Blecher CM. Clinical results and limitations of indirect decompression in spinal stenosis with laterally implanted interbody cages: results from a prospective cohort study. European spine journal : official publication of the European Spine Society, the European Spinal Deformity Society, and the European Section of the Cervical Spine Research Society. 2015;24 Suppl 3:339-45.

21. Alimi M, Lang G, Navarro-Ramirez R, Perrech M, Berlin C, Hofstetter CP, et al. The Impact of Cage Dimensions, Positioning, and Side of Approach in Extreme Lateral Interbody Fusion. Clinical spine surgery. 2018;31(1):E42-E9.

22. Tempel ZJ, McDowell MM, Panczykowski DM, Gandhoke GS, Hamilton DK, Okonkwo DO, et al. Graft subsidence as a predictor of revision surgery following stand-alone lateral lumbar interbody fusion. Journal of neurosurgery Spine. 2018;28(1):50-6.

23. Lam FC, Alkalay R, Groff MW. The effects of design and positioning of carbon fiber lumbar interbody cages and their subsidence in vertebral bodies. Journal of spinal disorders \& techniques. 2012;25(2):116-22. Epub 2011/03/25.

24. Navarro-Ramirez R, Lang G, Moriguchi Y, Elowitz E, Corredor JA, Avila MJ, et al. Are Locked Facets a Contraindication for Extreme Lateral Interbody Fusion? World neurosurgery. 2017;100:607-18.

25. Malham GM, Parker RM, Goss B, Blecher CM, Ballok ZE. Indirect foraminal decompression is independent of metabolically active facet arthropathy in extreme lateral interbody fusion. Spine. 2014;39(22):E1303-10.

26. Gabel BC, Hoshide R, Taylor W. An Algorithm to Predict Success of Indirect Decompression Using the Extreme Lateral Lumbar Interbody Fusion Procedure. Cureus. 2015;7(9):e317.

\section{Tables}


Table. 1 Demographic characteristics of patients with Schizas's classification D

\begin{tabular}{|ll|}
\hline Category & Values \\
\hline Average age & $67.94 \pm 4.21$ \\
\hline Gender(M:F) & $11: 7$ \\
\hline BMI in kg/m2 (mean) & $25.27 \pm 2.45$ \\
\hline Average surgical levels & $1.78 \pm 0.81$ \\
\hline Disc with Schizas grade D \\
\hline \multicolumn{1}{|c|}{ L3/4 } & 2 \\
\hline L4/5 & 16 \\
\hline VAS score for back & $6.06 \pm 1.35$ \\
\hline VAS score for leg & $5.39 \pm 1.24$ \\
\hline ODI score (\%) & $43.33 \pm 7.32$ \\
\hline Follow-up time(month) & $19.61 \pm 6.32$ \\
\hline
\end{tabular}


Table.2 Overall radiographic evaluation results

\begin{tabular}{|c|c|c|c|c|c|}
\hline Results $\square n=202 \square$ & preoperative & postoperative & Change & Change rate $\%$ & $P$ \\
\hline \multicolumn{6}{|l|}{ CT measurements } \\
\hline Anterior disk height & $10.74 \pm 3.25$ & $13.40 \pm 2.50$ & $2.66 \pm 2.08$ & $34.42 \pm 39.33$ & 00.01 \\
\hline Posterior disk height & $5.46 \pm 2.17$ & $7.70 \pm 2.34$ & $2.24 \pm 1.60$ & $58.30 \pm 64.68$ & $\square 0.01$ \\
\hline Disc angle & $5.80 \pm 3.38$ & $7.03 \pm 3.61$ & $1.23 \pm 3.81$ & - & 00.01 \\
\hline Left foraminal height & $17.70 \pm 2.33$ & $19.68 \pm 2.54$ & $1.98 \pm 1.82$ & $11.70 \pm 11.20$ & $\square 0.01$ \\
\hline Right foraminal height & $17.74 \pm 2.32$ & $19.92 \pm 2.48$ & $2.18 \pm 2.06$ & $13.12 \pm 13.08$ & 00.01 \\
\hline \multicolumn{6}{|l|}{ MRI measurements } \\
\hline Midsagittal canal diameter & $8.37 \pm 3.34$ & $10.36 \pm 3.15$ & $1.99 \pm 1.63$ & $35.86 \pm 64.01$ & 00.01 \\
\hline axial central canal area & $109.71 \pm 64.78$ & $130.57 \pm 66.83$ & $20.86 \pm 18.35$ & $25.97 \pm 26.06$ & 00.01 \\
\hline
\end{tabular}

This $P$ value is the result of comparison between before and after surgery. 
Page 14/23 


\section{Table.3 Summary of MRI evaluation}

\begin{tabular}{|c|c|c|c|c|c|c|}
\hline Parameter & Grade & preoperative & postoperative & Change value & Change rate \% & $P$ \\
\hline \multirow[t]{4}{*}{ Midsagittal canal diameter } & A & $11.50 \pm 3.78$ & $12.76 \pm 3.67$ & $1.26 \pm 1.21$ & $15.26 \pm 26.19$ & $\square 0.01$ \\
\hline & B & $9.34 \pm 2.52$ & $11.34 \pm 2.55$ & $2.00 \pm 1.67$ & $24.32 \pm 21.27$ & 0.21 \\
\hline & $\mathrm{C}$ & $7.38 \pm 2.19$ & $9.57 \pm 2.28$ & $2.17 \pm 1.75$ & $41.35 \pm 76.92$ & 0.34 \\
\hline & $\mathrm{D}$ & $4.39 \pm 2.52$ & $6.89 \pm 2.40$ & $2.50 \pm 1.25$ & $79.69 \pm 86.23$ & प \\
\hline \multirow[t]{4}{*}{ Axial central canal area } & A & $188.55 \pm 70.16$ & $211.79 \pm 69.20$ & $23.24 \pm 17.75$ & $14.51 \pm 13.30$ & 0.06 \\
\hline & B & $131.63 \pm 50.45$ & $150.66 \pm 49.65$ & $19.02 \pm 16.52$ & $17.41 \pm 15.52$ & 0.10 \\
\hline & $\mathrm{C}$ & $81.49 \pm 25.88$ & $103.08 \pm 31.96$ & $21.59 \pm 20.23$ & $29.96 \pm 27.90$ & 0.10 \\
\hline & $\mathrm{D}$ & $32.61 \pm 11.10$ & $49.72 \pm 17.47$ & $16.11 \pm 10.70$ & $52.91 \pm 34.41$ & प \\
\hline
\end{tabular}

This $P$ value is the result of comparison of change value with grade D. 
Page 16/23 
Table.4 Summary of CT evaluation

\begin{tabular}{|l|l|l|l|l|l|l|}
\hline Parameter & Grade & preoperative & postoperative & Change value & Change rate \% & $P$ \\
\hline Anterior disk height & A & $9.74 \pm 3.03$ & $12.89 \pm 2.38$ & $3.14 \pm 2.38$ & $41.23 \pm 39.32$ & 0.17 \\
\hline & B & $10.46 \pm 3.18$ & $13.34 \pm 2.25$ & $2.88 \pm 2.10$ & $37.55 \pm 43.48$ & 0.36 \\
\hline & C & $10.82 \pm 3.34$ & $13.27 \pm 2.61$ & $2.45 \pm 2.09$ & $31.46 \pm 40.32$ & 0.83 \\
\hline & D & $12.89 \pm 2.87$ & $15.22 \pm 2.34$ & $2.33 \pm 2.06$ & $21.39 \pm 20.73$ & - \\
\hline & A & $4.79 \pm 1.76$ & $7.48 \pm 1.77$ & $2.69 \pm 1.88$ & $75.18 \pm 72.83$ & 0.86 \\
\hline & B & $5.61 \pm 2.49$ & $8.24 \pm 2.34$ & $2.63 \pm 1.43$ & $66.69 \pm 68.34$ & 0.95 \\
\hline & C & $5.54 \pm 2.21$ & $7.38 \pm 2.34$ & $1.83 \pm 1.44$ & $47.11 \pm 58.30$ & 0.07 \\
\hline & D & $6.17 \pm 1.91$ & $8.83 \pm 1.95$ & $2.67 \pm 1.75$ & $55.97 \pm 57.35$ & - \\
\hline & $\mathrm{A}$ & $5.11 \pm 3.32$ & $6.85 \pm 2.74$ & $1.74 \pm 3.43$ & $\square$ & 0.48 \\
\hline & $\mathrm{B}$ & $5.09 \pm 3.41$ & $6.06 \pm 3.75$ & $0.96 \pm 3.85$ & $\square$ & 0.98 \\
\hline & $\mathrm{C}$ & $6.10 \pm 3.33$ & $7.33 \pm 3.84$ & $1.23 \pm 3.92$ & $\square$ & 0.81 \\
\hline & $\mathrm{D}$ & $6.74 \pm 3.63$ & $7.73 \pm 3.89$ & $0.99 \pm 3.89$ & $\square$ & - \\
\hline & $\mathrm{A}$ & $17.36 \pm 2.22$ & $19.86 \pm 2.43$ & $2.50 \pm 2.17$ & $15.31 \pm 14.52$ & 0.47 \\
\hline & $\mathrm{B}$ & $18.00 \pm 2.62$ & $20.40 \pm 2.88$ & $2.40 \pm 1.62$ & $13.75 \pm 9.26$ & 0.48 \\
\hline & $\mathrm{C}$ & $17.63 \pm 2.34$ & $19.18 \pm 2.46$ & $1.54 \pm 1.69$ & $9.28 \pm 10.01$ & 0.21 \\
\hline & $\mathrm{D}$ & $18.17 \pm 1.82$ & $20.28 \pm 1.60$ & $2.11 \pm 1.75$ & $12.20 \pm 10.28$ & - \\
\hline & $\mathrm{A}$ & $17.12 \pm 2.36$ & $19.88 \pm 2.17$ & $2.76 \pm 2.14$ & $17.29 \pm 13.43$ & 0.20 \\
\hline & $\mathrm{B}$ & $17.78 \pm 2.30$ & $20.67 \pm 2.71$ & $2.85 \pm 1.75$ & $16.70 \pm 12.51$ & 0.13 \\
\hline & $\mathrm{C}$ & $17.90 \pm 2.30$ & $19.54 \pm 2.52$ & $1.64 \pm 2.07$ & $9.90 \pm 12.88$ & 0.40 \\
\hline & $\mathrm{D}$ & $18.39 \pm 2.09$ & $20.44 \pm 2.15$ & $2.05 \pm 1.83$ & $11.83 \pm 10.82$ & - \\
\hline
\end{tabular}

This $P$ value is the result of comparison of change value with grade D.

Figures 
Figure.1

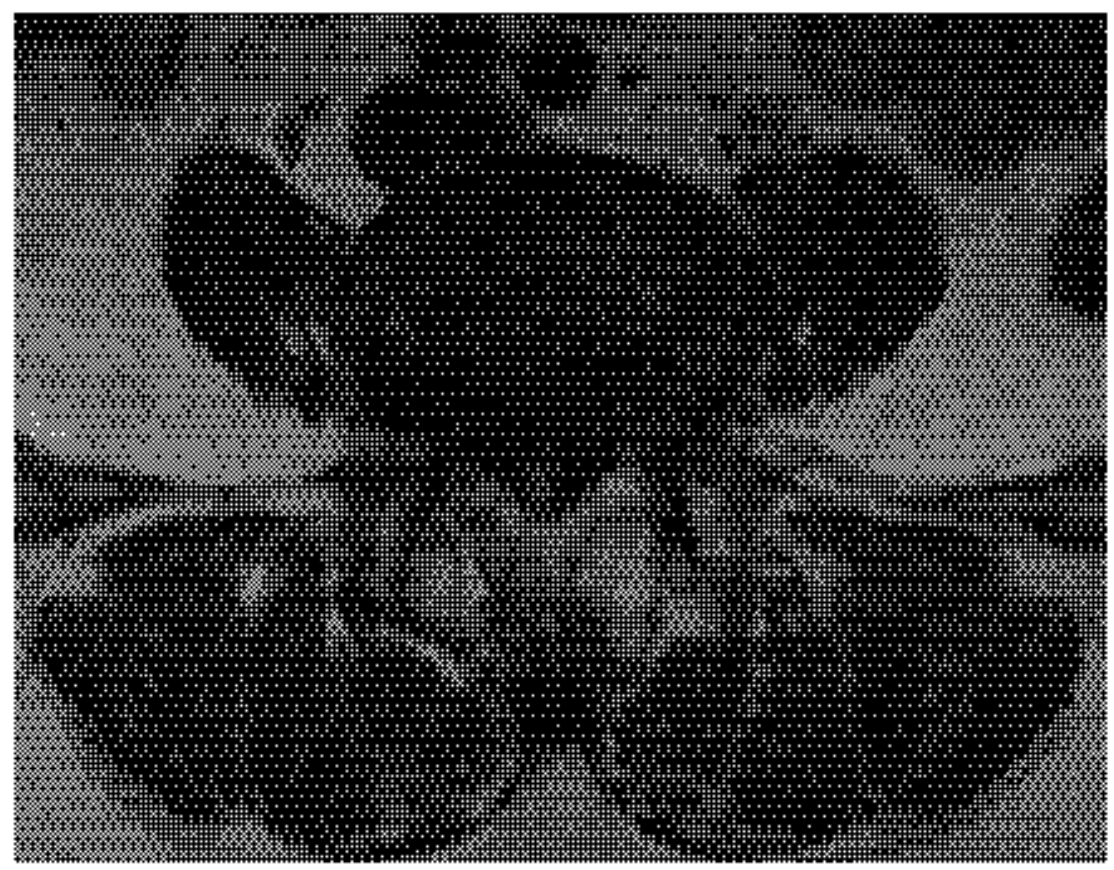

Figure 1

Extreme lumbar stenosis $₫$ Schizas stenosis grade $D$ 
Figure. 2
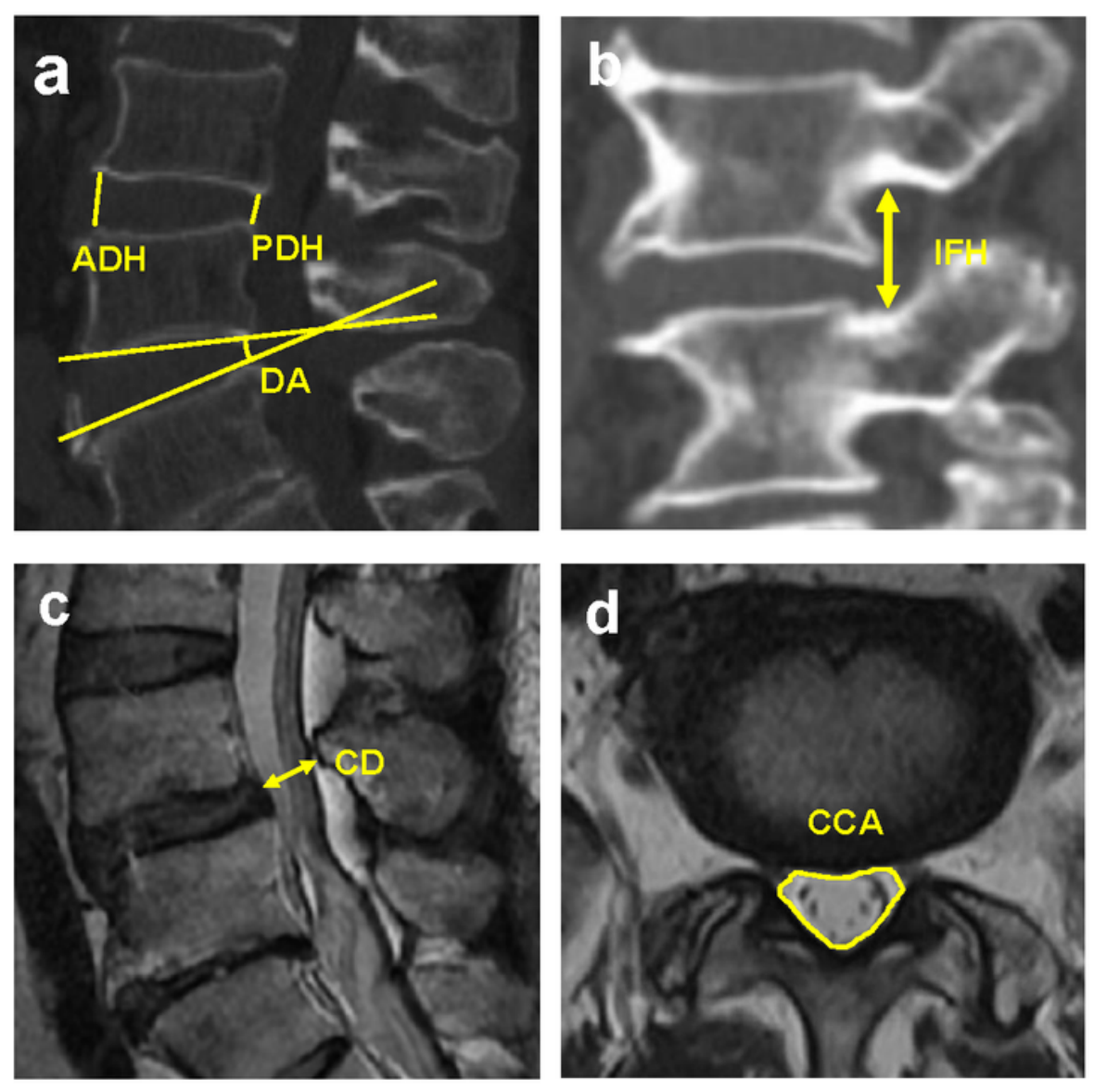

Figure 2

Measurement of radiographic parameters. ADH: anterior disc height. PDH: posterior disc height. DA: disc angle. IFH: intervertebral foramen height. CD: midsagittal canal diameter. CCA: axial central canal area. 
Figure.3

a

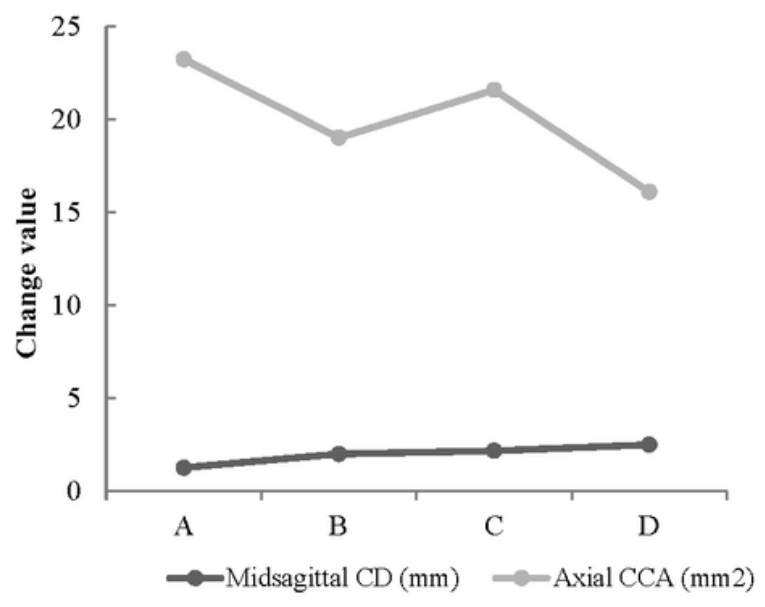

b

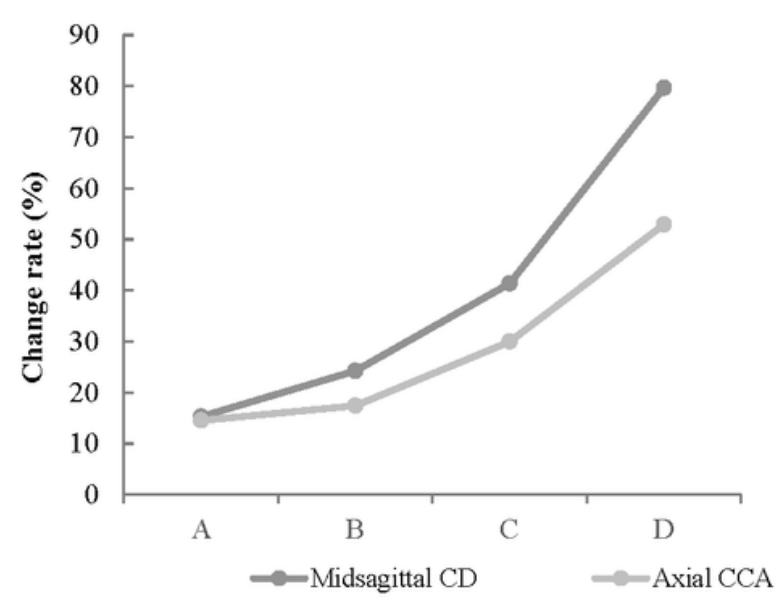

\section{Figure 3}

Change of spinal canal on MRI according to Schizas' classification. The average change of midsagittal $C D$ of grade $D$ was significantly greater than grade $A$, but showed no significant difference compared to grade $B$ or $C$. The average change of axial CCA of grade D was not significantly different from the others. However, the average change rate of midsagittal CD and axial CCA increased from grade A to grade $D$. 


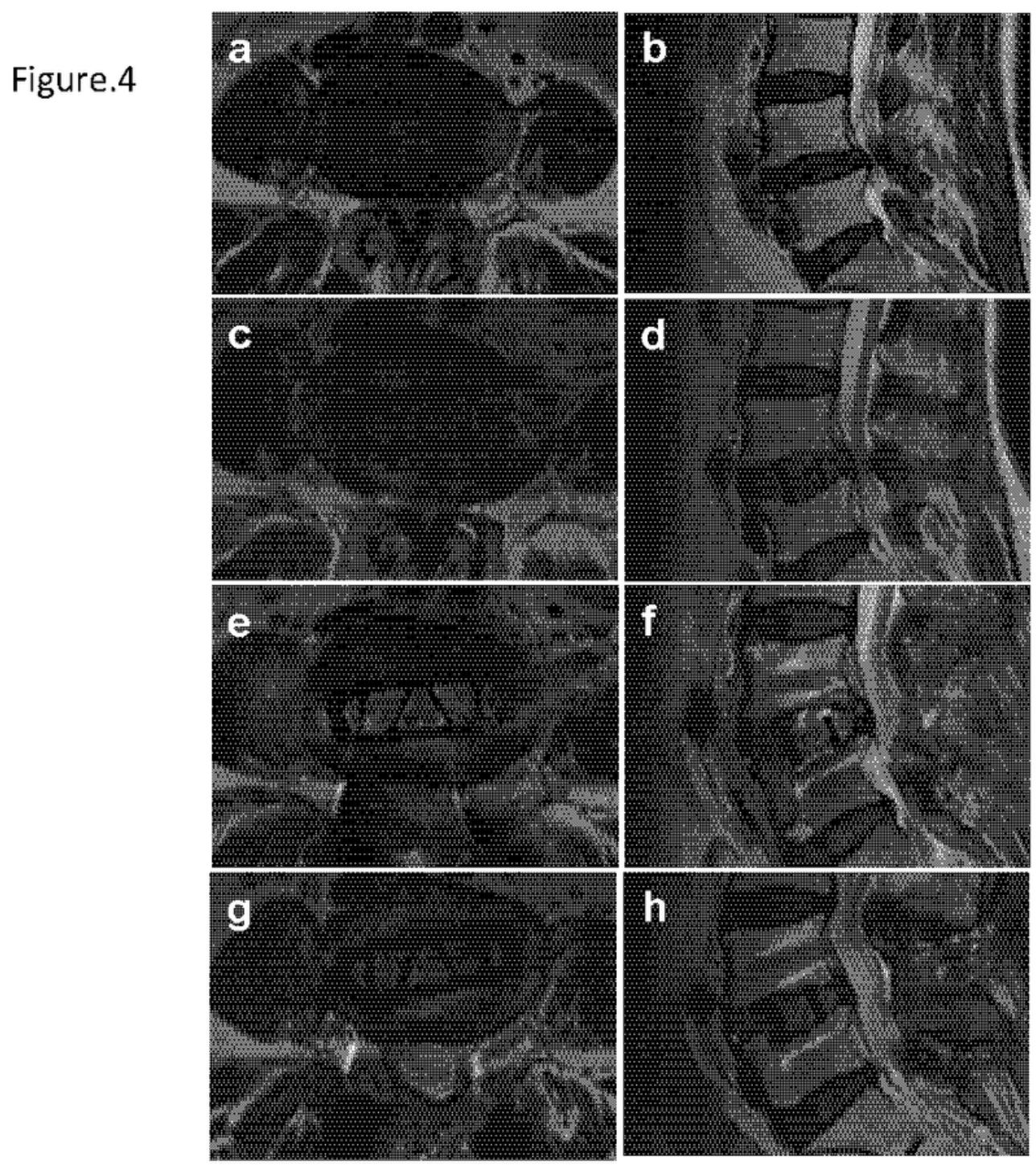

\section{Figure 4}

A 66-year-old woman with Schizas grade D preoperatively at $L 4 / 5(a, b)$. Her axial central canal area and midsagittal canal diameter partially improved after LLIF surgery (c, d) and significantly improved after second-stage laminectomy $(e, f)$. Neurological decompression was maintained 15 months after surgery $(\mathrm{g}, \mathrm{h})$. 
Figure.5
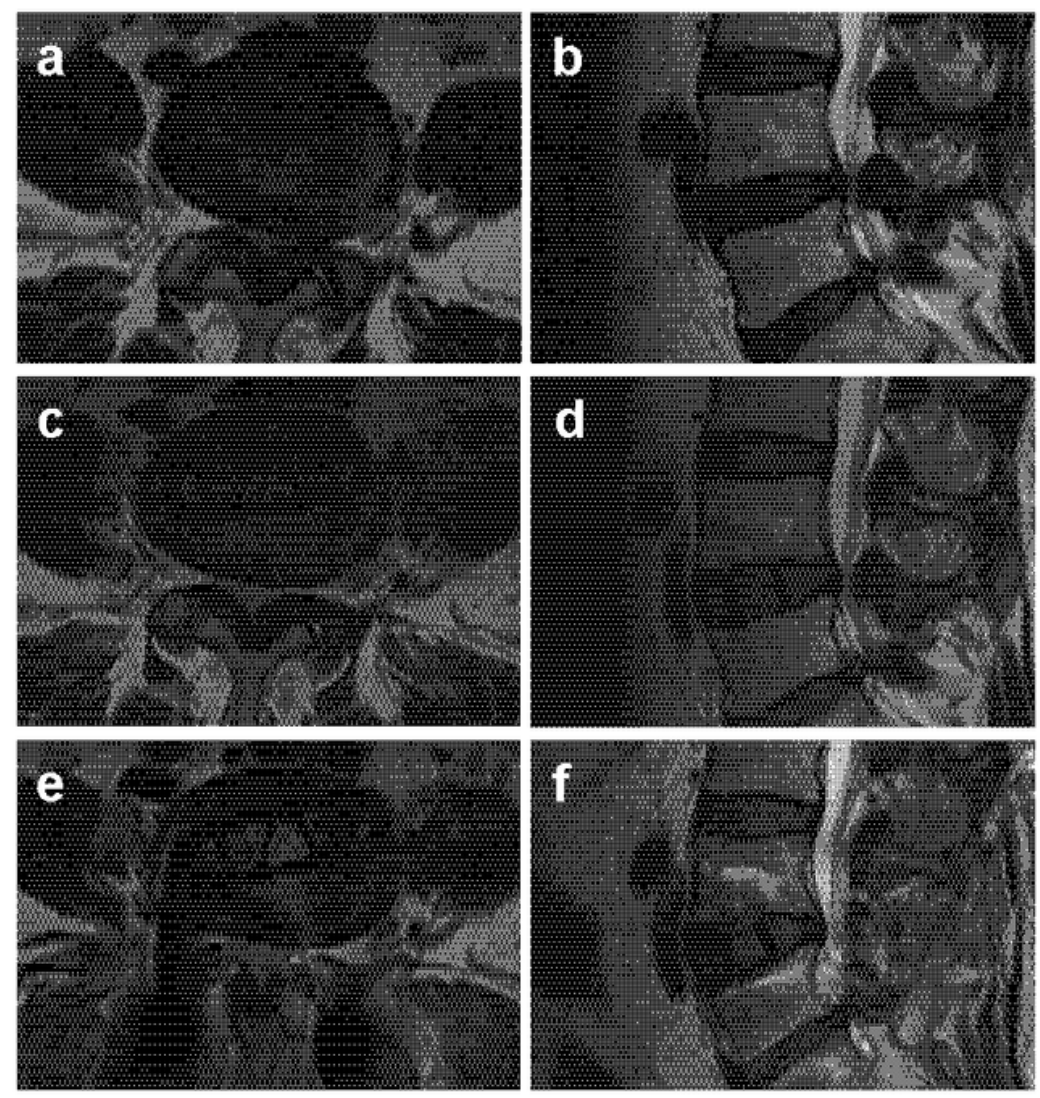

Figure 5

A 70-year-old woman with Schizas grade $D$ and severe ligamentum flavum hypertrophy preoperatively at L4/5 $(\mathrm{a}, \mathrm{b})$. Her axial central canal area and midsagittal canal diameter achieved small improvements after LLIF surgery with the presence of ligamentum flavum hypertrophy (c, d). Significant improvement was achieved after second-stage laminectomy $(e, f)$. 
Figure.6

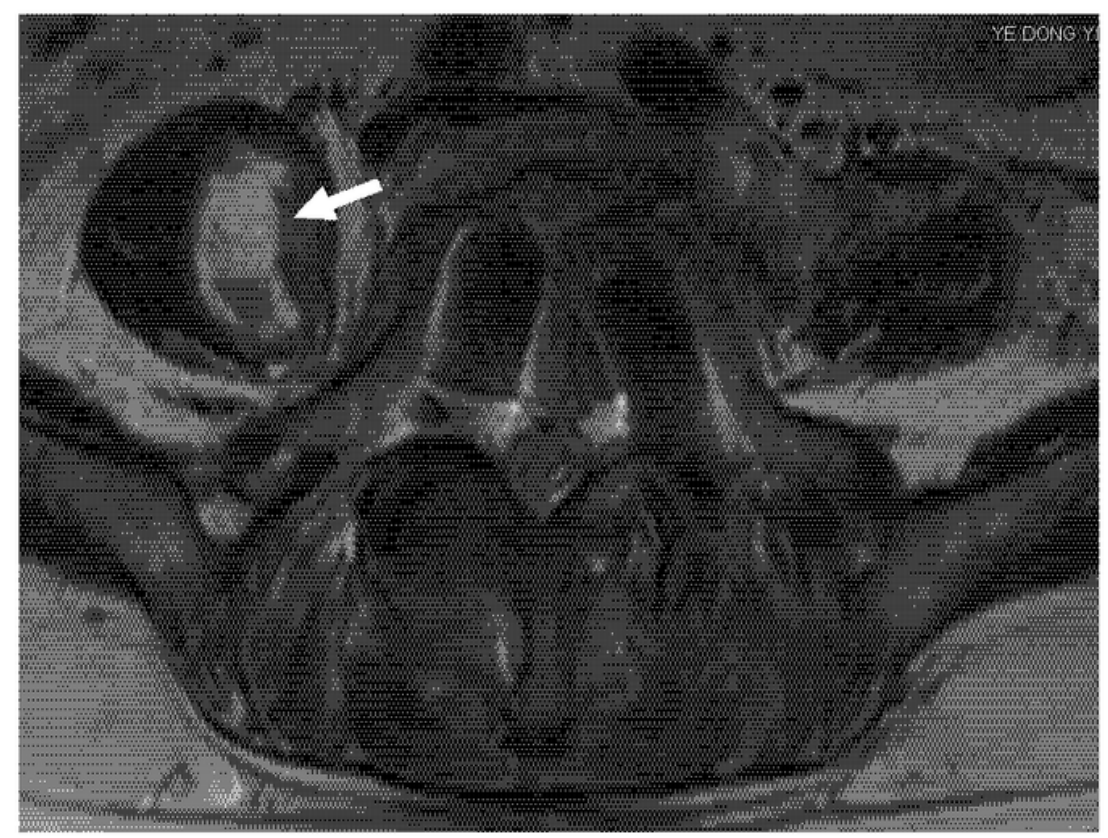

Figure 6

A 66-year-old woman suffered from contralateral iliopsoas hematoma (arrow). 